8 Migliori GB, Sotgiu G, Gandhi NR, et al. Drug resistance beyond extensively drug-resistant tuberculosis: individual patient data meta-analysis. Eur Respir J 2013; 42: 169-179.

9 Migliori GB, De Iaco G, Besozzi G, et al. First tuberculosis cases in Italy resistant to all tested drugs. Euro Surveill 2007; 12: E070517.1.

10 Matteelli A, D’Ambrosio L, Centis R, et al. Compassionate and optimum use of new tuberculosis drugs. Lancet Infect Dis 2015; 15: 1131-1132.

11 World Health Organization. The use of delamanid in the treatment of multidrug-resistant tuberculosis. Interim policy guidance. WHO/HTM/TB2014.23. Geneva, World Health Organization, 2014.

12 World Health Organization. The use of bedaquiline in the treatment of multidrug-resistant tuberculosis. Interim policy guidance. WHO/HTM/TB/2013.6. Geneva, World Health Organization, 2013.

13 Migliori GB, Lienhardt $\mathrm{C}$, Weyer $\mathrm{K}$, et al. Ensuring rational introduction and responsible use of new TB tools: outcome of an ERS multisector consultation. Eur Respir J 2014; 44: 1412-1417.

14 Srikrishna G, Gupta S, Dooley KE, et al. Can the addition of verapamil to bedaquiline-containing regimens improve tuberculosis treatment outcomes? A novel approach to optimizing TB treatment. Future Microbiol 2015; 10: $1257-1260$.

15 Guglielmetti L, Le Dû D, Jachym M, et al. Compassionate use of bedaquiline for the treatment of multidrug-resistant and extensively drug-resistant tuberculosis: interim analysis of a French cohort. Clin Infect Dis 2015; 60: 188-194.

\title{
Compassionate use of new drugs in children and adolescents with multidrug-resistant and extensively drug-resistant tuberculosis: early experiences and challenges
}

To the Editor:

The World Health Organization (WHO) estimated that 480000 new multidrug-resistant (MDR) tuberculosis (TB) cases occurred globally in 2014, with 190000 deaths. Limited data are available on the burden of MDR-TB in children. A recent systematic review estimated that 32000 children acquire MDR-TB annually; of these, very few are correctly diagnosed and provided with appropriate treatment [1].

Treatment of drug-resistant TB is long, expensive and associated with frequent adverse events [1-5]. In children, treatment is further complicated by limited data on appropriate dosing and safety, and a lack of child-friendly formulations. New anti-TB drugs are urgently needed to improve treatment tolerability and outcome, particularly for MDR-TB cases with additional second-line drug resistance, for whom identifying at least four active drugs is difficult with the current armamentarium of drugs [1, 4-11]. Two novel anti-TB drugs, delamanid $[6,8,12]$ and bedaquiline $[6,7,9,10,12]$, have received conditional approval for use in adults with MDR-TB. While the WHO interim guidance does not include a recommendation on their use in children due to lack of data, the Centers for Disease Control and Prevention guidelines state that bedaquiline use can be considered for children when treatment options are limited.

A number of paediatric clinical trials are ongoing or planned. The Otsuka 232 (phase 1, pharmacokinetics and safety to determine the appropriate dose for MDR-TB in children) and 233 (phase 2, 6-month safety, efficacy and pharmacokinetics trial in children with MDR-TB) trials of delamanid have begun recruitment. Preliminary pharmacokinetics and safety data in 6-17-year-old children from these trials have been presented, showing an excellent safety profile and leading to weight-based dosing recommendations for patients of $20-35 \mathrm{~kg}$ to receive half the adult dose (50 mg of delamanid twice daily) (presented in poster format (A-960) by J. Hafkin; Pharmacokinetics and safety of delamanid in paediatric MDR-TB patients, ages 6-17 years; San Diego, Interscience Conference on Antimicrobial Agents and Chemotherapy (ICAAC), 2015). A follow-up study to confirm the long-term safety, tolerability and pharmacokinetics of delamanid in these age groups and younger children (0-5 years) is ongoing and data are not yet available (presented in poster format (EP-115-04) by J. Hafkin; Long-term safety, tolerability and pharmacokinetics of delamanid in paediatric MDR-TB patients, ages 12-17 years; Cape Town, The 46th Union World Conference on Lung 
Health, 2015). Neither the Janssen C21 trial of bedaquiline (phase 2, pharmacokinetics, tolerability and anti-mycobacterial activity in children with MDR-TB) nor the US National Institutes of Health International Maternal Pediatric Adolescent AIDS Clinical Trials (IMPAACT) network planned trial of bedaquiline pharmacokinetics and safety in HIV-infected and uninfected children, have yet begun enrolling.

The Janssen bedaquiline compassionate use programme excluded all subjects aged <18 years, while delamanid is available to children from 12 years of age, and recently from 6 years, through the Otsuka compassionate use programme. Each request for delamanid compassionate use submitted to Otsuka is reviewed by an independent panel of experts, to assess the appropriateness and safety of delamanid use before the drug is provided. The aim of this report is to share the initial experience and challenges of compassionate use of delamanid in children and adolescents at a global level. The information reported here was collected through the two bodies providing independent advice on the rational use of new drugs: the TB Consilium (www.tbconsilium.org) [8] and the endTB committee (Médecins Sans Frontières (MSF), Partners in Health, and Interactive Research and Development; www.endtb.org).

Between February 2014 and March 2016, Otsuka received 19 requests for delamanid compassionate use for paediatric patients aged $<18$ years. All requests were considered appropriate by both the external committees and Otsuka, and hence were enrolled in the delamanid compassionate use programme. Requests were received from Italy $(n=1)$, South Africa $(n=5)$, Georgia (MSF settings; $n=4)$, India $(n=5$; four from MSF settings), Namibia ( $n=1$ ), Swaziland, Russia and Armenia (MSF settings; one case each). Data from patients are summarised in table 1.

The rationale for delamanid requests was mainly the limited treatment options due to extensive resistance pattern or poor response to second-line anti-TB treatment, although in one case it was for reinforcement of the treatment regimen in a patient with adverse events. The TB Consilium and endTB committee experts recommended the use of delamanid as the best treatment option in these patients.

All 19 paediatric cases enrolled in the delamanid compassionate use programme had bacteriologically confirmed pulmonary MDR- or extensively drug-resistant (XDR)-TB (two cases also had extrapulmonary TB). Drug susceptibility testing confirmed resistance to four to 15 anti-TB drugs. Only three patients (patients 5, 6 and 19 in table 1) were HIV positive.

Out of 19 enrolled patients, 16 (84\%) have already started treatment with delamanid. The mean age at treatment initiation was 14.4 years (range 8-17 years). All cases received delamanid $100 \mathrm{mg}$ twice daily (adult dosage), except one who received $50 \mathrm{mg}$ twice daily due to a body weight of $22 \mathrm{~kg}$. Of these, six patients have already completed 24 weeks of delamanid, while 10 are still receiving the delamanid.

All patients showed good tolerability to delamanid with no or mild adverse events, except one patient from India. This patient was receiving a combination of delamanid-capreomycin-ethionamide-cycloserineclofazimine-imipenem-amoxicillin/clavulanate-pyrazinamide, and experienced severe vomiting, renal impairment and severe electrolyte disturbances (hypokalaemia and hypomagnesaemia) that led to QTcF (QT interval in the ECG corrected according to Fredericia formula) prolongation ( $>500 \mathrm{~ms}$ ) requiring temporary delamanid discontinuation (albumin was normal). After management of vomiting and electrolyte imbalance correction, the patient was able to complete delamanid treatment without further QTcF prolongation.

As shown in table 1, the interim treatment response is good: 13 (81.2\%) out of 16 were Mycobacterium tuberculosis culture-negative at the time of this report (three patients were recently started on delamanid, so the interim treatment responses are not yet available). Except for one patient who has successfully completed MDR-TB treatment, the remaining patients are continuing treatment and do not have final treatment outcomes yet.

The combination of delamanid and bedaquiline has been limited due to concerns regarding the QT prolongation effects of both drugs. However, there are initial experiences with their simultaneous use in adults (now also possible through compassionate use) $[13,14]$. This combination could be considered in children in the absence of effective alternative treatment options $[4,5]$, under enhanced clinical monitoring [12-15].

Globally, there are very few children who have access to delamanid compared with the likely number who could benefit from it. Healthcare providers have described a number of challenges to the access of delamanid. The compassionate use mechanism, despite efforts to facilitate quick access, is a long, multistep and time-demanding process (request, permission for importation/use, informed consent, health staff training on the compassionate use protocol, reporting requirements and importation). This can result in substantial delays in treatment initiation, which could adversely affect treatment outcome. In our study, the median delay between process initiation and delamanid initiation was 73.5 days (range 14-153 days). 
TABLE 1 Paediatric cases enrolled in Otsuka delamanid compassionate use programme, from February 2014 to March 2016

\begin{tabular}{|c|c|c|c|c|c|c|c|c|c|c|}
\hline Patient & $\begin{array}{l}\text { Country } \\
\text { of origin }\end{array}$ & $\begin{array}{l}\text { Age } \\
\text { years }\end{array}$ & Sex & TB form & $\begin{array}{l}\text { Test results at time of } \\
\text { delamanid request }\end{array}$ & Resistance profile & $\begin{array}{l}\text { Expert panel consulted and } \\
\text { indication for delamanid }\end{array}$ & $\begin{array}{l}\text { Drugs used prior to } \\
\text { delamanid }\end{array}$ & $\begin{array}{l}\text { Started } \\
\text { delamanid }\end{array}$ & $\begin{array}{l}\text { Treatment outcome or } \\
\text { interim treatment response }\end{array}$ \\
\hline 1 & Italy & 13 & M & $P$ and $E P$ & SS+/C+ (MGIT), Xpert+ & $\begin{array}{c}\text { XDR-TB: H, R, Z, E, S, } \\
\text { Rfb, Amk, Cm, Km, } \\
\text { Lfx, Mfx, Ofx, Eto, Pto, } \\
\text { HdH }\end{array}$ & $\begin{array}{l}\text { TB Consilium: extensive } \\
\text { resistance to SLDs, drug } \\
\text { toxicity and limited options for } \\
\text { treatment }\end{array}$ & $\begin{array}{c}\text { H, Z, E, Amk, Mfx, Eto, } \\
\text { PAS, Tzd, Amx/Clv, Clr, } \\
\text { Cfz, Lzd, Mpm }\end{array}$ & Yes & Cured \\
\hline 2 & $\begin{array}{l}\text { South } \\
\text { Africa }\end{array}$ & 17 & $\mathrm{~F}$ & $P$ & SS+/C+, Xpert+ & $\begin{array}{l}\text { XDR-TB: H, R, Amk, } \\
\text { Km, Ofx, Eto }\end{array}$ & $\begin{array}{l}\text { TB Consilium: extensive } \\
\text { resistance to SLDs and severe } \\
\text { clinical presentation }\end{array}$ & $\begin{array}{l}\text { H, Z, E, Cm, Km, Mfx, } \\
\text { Eto, PAS, Tzd, Cfz, HdH }\end{array}$ & $\mathrm{No}^{\#}$ & \\
\hline 3 & $\begin{array}{l}\text { South } \\
\text { Africa }\end{array}$ & 13 & M & $P$ & SS+/C+, Xpert+ & $\begin{array}{c}\text { XDR-TB: H, R, Amk, } \\
\text { Ofx }\end{array}$ & $\begin{array}{l}\text { TB Consilium: extensive } \\
\text { resistance to SLDs and severe } \\
\text { clinical presentation }\end{array}$ & $\begin{array}{c}\text { Z, E, Cm, Km, Mfx, Eto, } \\
\text { PAS, Tzd, Amx/Clv, Clr, } \\
\text { Cfz, Lzd }\end{array}$ & Yes & $\begin{array}{l}\text { Currently culture negative, } \\
\text { good clinical response, } \\
\text { delamanid completed }\end{array}$ \\
\hline 4 & $\begin{array}{l}\text { South } \\
\text { Africa }\end{array}$ & 13 & $\mathrm{~F}$ & $P$ & SS-/C+ (MGIT), Xpert+ & $\begin{array}{c}\text { XDR-TB: H, R, Amk, } \\
\text { Ofx }\end{array}$ & $\begin{array}{l}\text { TB Consilium: extensive } \\
\text { resistance to SLDs and severe } \\
\text { clinical presentation }\end{array}$ & $\begin{array}{l}\text { E, Z, Cm, Mfx, Eto, PAS, } \\
\text { Tzd, Cfz, HdH, Lzd }\end{array}$ & Yes & $\begin{array}{l}\text { Currently culture negative, } \\
\text { good clinical response, } \\
\text { delamanid ongoing }\end{array}$ \\
\hline 5 & $\begin{array}{l}\text { South } \\
\text { Africa }\end{array}$ & 8 & M & $P$ & $\begin{array}{l}\text { SS+/C+ (MGIT), Xpert } \\
\text { MTB+, R resistant }\end{array}$ & $\begin{array}{l}\text { Pre-XDR: H, R, Amk, } \\
\text { Cm, Km, Eto }\end{array}$ & $\begin{array}{l}\text { TB Consilium: extensive } \\
\text { resistance to SLDs and severe } \\
\text { clinical presentation }\end{array}$ & Z, Amk, Mfx, Eto, Tzd & Yes & $\begin{array}{c}\text { First culture not yet } \\
\text { available }\end{array}$ \\
\hline 6 & Namibia & 9 & M & $P$ and $E P$ & $\begin{array}{l}\text { SS-/C-, lymph C+ } \\
\text { (MGIT) }\end{array}$ & $\begin{array}{l}\text { XDR-TB: H, R, E, S, } \\
\text { Amk, Cm, Km, Lfx, } \\
\text { Mfx, Ofx, Eto, PAS, } \\
\text { Cs, HdH }\end{array}$ & $\begin{array}{l}\text { TB Consilium: extensive } \\
\text { resistance to SLDs and severe } \\
\text { clinical presentation }\end{array}$ & $\begin{array}{l}\text { Z, Cm, Mfx, PAS, Cs, } \\
\text { Amx/Clv, Clr, Cfz, HdH }\end{array}$ & Noף & \\
\hline 7 & $\begin{array}{l}\text { South } \\
\text { Africa }\end{array}$ & 12 & $\mathrm{~F}$ & $P$ & $\begin{array}{l}\text { SS-/C+ (MGIT), Xpert } \\
\text { indeterminate }\end{array}$ & $\begin{array}{l}\text { XDR-TB: H, R, S, } \\
\text { Amk, Cm, Km, Ofx, } \\
\text { Eto, Pto, HdH }\end{array}$ & $\begin{array}{l}\text { TB Consilium: extensive } \\
\text { resistance to SLDs and severe } \\
\text { clinical presentation }\end{array}$ & $H, R, Z, E, E t o, T z d$, Lfx & Yes & $\begin{array}{l}\text { Currently culture negative, } \\
\text { good clinical response, } \\
\text { delamanid ongoing }\end{array}$ \\
\hline 8 & India & 12 & $\mathrm{~F}$ & $P$ & $\mathrm{SS}+/ \mathrm{C}+(\mathrm{MGIT})$ & $\begin{array}{l}\text { XDR-TB: R, H, Z, E, S, } \\
\text { Amk, Cm, Km, Mfx, } \\
\text { Ofx, Eto, PAS, Cs, Lzd }\end{array}$ & $\begin{array}{l}\text { TB Consilium: extensive } \\
\text { resistance to SLDs and severe } \\
\text { clinical presentation }\end{array}$ & $\begin{array}{c}\text { E, Z, Cm, Mfx, PAS, Cs, } \\
\text { Amx/Clv, Clr, Cfz, Lzd, } \\
\text { Mpm }\end{array}$ & Yes & $\begin{array}{c}\text { First culture not yet } \\
\text { available }\end{array}$ \\
\hline 9 & India & 17 & $\mathrm{~F}$ & $P$ & $\mathrm{SS}+\mathrm{C}+(\mathrm{MGIT})$ & $\begin{array}{l}\text { XDR-TB: H, R, E, S, } \\
\text { Amk, Cm, Km, Mfx, } \\
\text { Ofx, Eto, PAS }\end{array}$ & $\begin{array}{l}\text { endTB committee: extensive } \\
\text { resistance to SLDs }\end{array}$ & $\begin{array}{c}\text { H, R, Z, E, S, Km, Mfx, } \\
\text { Pto, PAS, Cs, Amx/Clv, } \\
\text { Clr, Cfz, Lzd }\end{array}$ & Yes & $\begin{array}{l}\text { Currently culture negative, } \\
\text { good clinical response, } \\
\text { delamanid completed }\end{array}$ \\
\hline 10 & India & 15 & $\mathrm{~F}$ & $P$ & $\mathrm{SS}+/ \mathrm{C}+(\mathrm{MGIT})$ & $\begin{array}{c}\text { XDR-TB: H, R, E, S, } \\
\text { Amk, Km, Ofx, Eto, } \\
\text { PAS, Cfz }\end{array}$ & $\begin{array}{l}\text { endTB committee: extensive } \\
\text { resistance to SLDs }\end{array}$ & $\begin{array}{c}H, R, Z, E, K m, M f x, \text { Eto, } \\
\text { PAS, Cfz, Lzd }\end{array}$ & Yes & $\begin{array}{l}\text { Currently culture negative, } \\
\text { good clinical response, } \\
\text { delamanid completed }\end{array}$ \\
\hline 11 & India & 16 & M & $P$ & SS-/C+ (MGIT) & $\begin{array}{l}\text { XDR-TB: H, R, E, S, } \\
\text { Amk, Cm, Km, Mfx, } \\
\text { Ofx, Eto, PAS }\end{array}$ & $\begin{array}{l}\text { endTB committee: failure of } \\
\text { previous treatment and } \\
\text { extensive resistance to SLDs }\end{array}$ & $\begin{array}{l}\text { H, R, Z, E, Rfb, Cm, Km, } \\
\text { Lfx, Mfx, Eto, PAS, Cs, } \\
\text { Amx/Clv, Cfz, HdH, Lzd }\end{array}$ & Yes & $\begin{array}{l}\text { Culture negative, good } \\
\text { clinical response, } \\
\text { delamanid completed }\end{array}$ \\
\hline 12 & India & 13 & $\mathrm{~F}$ & $\begin{array}{l}\text { P and EP } \\
\text { (lymph } \\
\text { node) }\end{array}$ & $\begin{array}{c}\text { SS+/C+ (MGIT), Xpert } \\
\text { MTB+, R resistant }\end{array}$ & $\begin{array}{l}\text { XDR-TB: H, R, E, Z, S, } \\
\text { Amk, Cm, Km, Mfx, } \\
\text { Ofx, Eto, PAS, Cs }\end{array}$ & $\begin{array}{l}\text { endTB committee: extensive } \\
\text { resistance to SLDs }\end{array}$ & $H, R, Z, E$ & $\mathrm{No}^{+}$ & \\
\hline 13 & Georgia & 16 & M & $\mathrm{P}$ & $\begin{array}{c}\text { SS+/C+ (MGIT), Xpert } \\
\text { MTB+, R resistant }\end{array}$ & MDR-TB: H, R, E, Z & $\begin{array}{l}\text { endTB committee: no } \\
\text { improvement with SLDs (still } \\
\text { smear positive after } 3 \text { months } \\
\text { of treatment) }\end{array}$ & $\begin{array}{c}\mathrm{E}, \mathrm{Z}, \mathrm{Cm}, \mathrm{Mfx}, \mathrm{Cs}, \mathrm{PAS}, \\
\text { Lzd }\end{array}$ & Yes & $\begin{array}{l}\text { Currently culture negative, } \\
\text { good clinical response, } \\
\text { delamanid ongoing }\end{array}$ \\
\hline
\end{tabular}




\begin{tabular}{|c|c|c|c|c|c|c|c|c|c|c|}
\hline Patient & $\begin{array}{l}\text { Country } \\
\text { of origin }\end{array}$ & $\begin{array}{l}\text { Age } \\
\text { years }\end{array}$ & Sex & TB form & $\begin{array}{l}\text { Test results at time of } \\
\text { delamanid request }\end{array}$ & Resistance profile & $\begin{array}{l}\text { Expert panel consulted and } \\
\text { indication for delamanid }\end{array}$ & $\begin{array}{l}\text { Drugs used prior to } \\
\text { delamanid }\end{array}$ & $\begin{array}{l}\text { Started } \\
\text { delamanid }\end{array}$ & $\begin{array}{l}\text { Treatment outcome or } \\
\text { interim treatment response }\end{array}$ \\
\hline 14 & Georgia & 17 & M & $P$ & $\begin{array}{l}\text { SS-/C+ (MGIT), Xpert } \\
\text { MTB+, R resistant }\end{array}$ & $\begin{array}{c}\text { Pre-XDR (FQ): H, R, E, } \\
\text { S, Ofx }\end{array}$ & $\begin{array}{l}\text { endTB committee: no } \\
\text { improvement with SLDs } \\
\text { (culture positive after } 7 \text { months } \\
\text { of treatment, progression of } \\
\text { infiltrates and cavities) }\end{array}$ & $\begin{array}{l}H, Z, E, C m, \text { Lfx, Mfx } \\
\text { Pto, PAS, Cs }\end{array}$ & Yes & $\begin{array}{l}\text { Culture negative, good } \\
\text { clinical response, } \\
\text { delamanid ongoing }\end{array}$ \\
\hline 15 & Georgia & 15 & M & $P$ & $\begin{array}{l}\text { SS-/C+ (MGIT), HAIN } \\
\text { MTB+, R resistant }\end{array}$ & $\begin{array}{c}\text { XDR-TB: H, R, E, S, } \\
\text { Km, Ofx }\end{array}$ & $\begin{array}{l}\text { endTB committee: extensive } \\
\text { resistance to SLDs and severe } \\
\text { clinical presentation (bilateral } \\
\text { (esions) }\end{array}$ & $\begin{array}{l}\text { H, R, Z, E, Cm, Lfx, Mfx, } \\
\text { Pto, PAS, Cs, Cfz, Lzd }\end{array}$ & Yes & $\begin{array}{l}\text { Culture negative, good } \\
\text { clinical response, } \\
\text { delamanid completed }\end{array}$ \\
\hline 16 & Georgia & 17 & M & $P$ & $\begin{array}{c}\text { SS+/C+ (MGIT), Xpert } \\
\text { MTB+, R resistant }\end{array}$ & $\begin{array}{c}\text { Pre-XDR (FQ): H, R, E, } \\
\text { S, Ofx }\end{array}$ & $\begin{array}{l}\text { endTB committee: no clinical or } \\
\text { radiological improvement with } \\
\text { SLDs }\end{array}$ & $\begin{array}{c}\text { E, Z, Cm, Lfx, Mfx, Pto, } \\
\text { PAS, Cs, Amx/Clv, Clr, } \\
\text { Cfz }\end{array}$ & Yes & $\begin{array}{l}\text { Culture negative, good } \\
\text { clinical response, } \\
\text { delamanid completed }\end{array}$ \\
\hline 17 & Russia & 16 & $\mathrm{~F}$ & $P$ & $\begin{array}{c}\text { SS+/C+ (MGIT), Xpert } \\
\text { MTB+, R resistant }\end{array}$ & $\begin{array}{c}\text { XDR-TB: } \mathrm{H}, \mathrm{R}, \mathrm{S}, \mathrm{Cm}, \\
\text { Lfx }\end{array}$ & $\begin{array}{c}\text { endTB committee: extensive } \\
\text { resistance to SLDs and severe } \\
\text { clinical presentation (bilateral } \\
\text { (esions) }\end{array}$ & Z, Cm, Lfx, Pto, PAS, Cs & Yes & $\begin{array}{l}\text { Currently culture negative, } \\
\text { good clinical response, } \\
\text { delamanid ongoing }\end{array}$ \\
\hline 18 & Armenia & 16 & M & $P$ & $\begin{array}{c}\text { SS+/C+ (MGIT), Xpert } \\
\text { MTB+, R resistant }\end{array}$ & $\begin{array}{l}\text { Pre-XDR (FQ): H, R, Z, } \\
\text { E, Amk, Cm, Pto }\end{array}$ & $\begin{array}{l}\text { endTB committee: extensive } \\
\text { resistance to SLDs and drug } \\
\text { toxicity }\end{array}$ & $\begin{array}{l}\text { Cm, Lfx, Pto, PAS, Cs, } \\
\text { Amx/Clv, Cfz }\end{array}$ & Yes & $\begin{array}{l}\text { Currently culture negative, } \\
\text { good clinical response, } \\
\text { delamanid ongoing }\end{array}$ \\
\hline 19 & Swaziland & 15 & M & $P$ & $\begin{array}{c}\text { SS+/C+ (MGIT), Xpert } \\
\text { MTB+, R resistant }\end{array}$ & $\begin{array}{l}\text { XDR-TB: H, R, E, S, } \\
\text { Cm, Km, Mfx }\end{array}$ & $\begin{array}{l}\text { endTB committee: failure of } \\
\text { previous treatment and } \\
\text { extensive resistance to SLDs }\end{array}$ & Z, Km, Lfx, Pto, PAS, Cs & Yes & $\begin{array}{c}\text { First culture not yet } \\
\text { available }\end{array}$ \\
\hline
\end{tabular}

TB: tuberculosis; SS: sputum smear; C: culture; M: male; F: female; P: pulmonary; EP: extrapulmonary; MGIT: Mycobacteria Growth Indicator Tube (Becton, Dickinson and Company, (Cepheid, Sunnyvale, CA, USA); XDR: extensively drug-resistant; H: isoniazid; R: rifampicin; Z: pyrazinamide; E: ethambutol; S: streptomycin; Rfb: rifabutin; Amk: amikacin; Cm: capreomycin; Km: kanamycin; Lfx: levofloxacin; Mfx: moxifloxacin; Ofx: ofloxacin; Eto: ethionamide; Pto: prothionamide; $\mathrm{HdH}$ : high-dose isoniazid; SLDs: second-line drugs; PAS: para-aminosalicylic acid; Tzd: terizidone; Amx/Clv: amoxicillin plus clavulanate; Clr: clarithromycin; Cfz: clofazimine; Lzd: linezolid; Mpm: meropenem; Cs: cycloserine; MDR: multidrug-resistant; FQ: fluoroquinolones; HAIN: GenoType MTBDRplus (Hain Lifescience GmbH, Nehren, Germany). \#: after inclusion in delamanid compassionate use programme, treating clinicians decided to withdraw the request for delamanid for this patient due to adverse effects and poor treatment adherence; ${ }^{\text {? }}$ : awaiting import permit from the relevant authorities; ${ }^{+}$: patient withdrawn from compassionate use programme for programmatic reasons. 
The recent announcement that delamanid is accessible through the Global Drug Facility for USD 1700 per treatment is an important step forward in increasing international access, avoiding the need for a compassionate use mechanism. However, countries may still face other barriers, such as lack of appropriate tools (clinical guidelines, appropriate training and national implementation plans), pharmacovigilance reporting requirements (which, although essential, are a new challenge for providers and programmes), and, lastly, budgets to cover the costs of these drugs. All are required to ensure rational use of delamanid in adult and paediatric patients.

In addition, the lack of inclusion of children in bedaquiline trials to date is not acceptable and contributes to the current situation of very limited access to this drug among children.

Wider availability of both drugs for children, including those with clinical TB and contact with a known $\mathrm{MDR} / \mathrm{XDR}-\mathrm{TB}$ source case, is urgently needed, as is the proactive inclusion of children and adolescents in trials of novel TB drugs [16].

Case-by-case evaluation from independent bodies like the TB Consilium and endTB committee is a valuable support to clinicians and programmes in the use of the new drugs in children, particularly where the clinical cases are complex and in the absence of formal guidance for their paediatric use. The use of these services could contribute to an increase in the appropriate use of new drugs among children and adolescents in need.

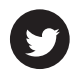

@ERSpublications

First experience and challenges of compassionate use of new anti-TB drugs to treat MDR- and XDR-TB in children http://ow.ly/SWXF300a0UX

Marina Tadolini ${ }^{1,21}$, Anthony J. Garcia-Prats ${ }^{2,21}$, Lia D’Ambrosio ${ }^{3,4,21}$, Catherine Hewison ${ }^{5,21}$, Rosella Centis ${ }^{3,21}$ H. Simon Schaaf ${ }^{2,21}$, Ben J. Marais ${ }^{6}$, Hannetjie Ferreira ${ }^{7}$, Jose A. Caminero ${ }^{8,9}$, Sylvie Jonckheere ${ }^{10}$, Animesh Sinha ${ }^{11}$, Krzysztof Herboczek ${ }^{12}$, Zarema Khaidarkhanova ${ }^{13}$, Armen Hayrapetyan ${ }^{14}$, Naira Khachatryan ${ }^{15}$, Ia Urtkmelidze ${ }^{16}$, Carolina Loreti ${ }^{17}$, Susanna Esposito ${ }^{18}$, Alberto Matteelli ${ }^{19}$, Jennifer Furin ${ }^{20}$, Francis Varaine ${ }^{5}$ and Giovanni Battista Migliori (10 ${ }^{3}$

${ }^{1}$ Unit of Infectious Diseases, Dept of Medical and Surgical Sciences, Alma Mater Studiorum University of Bologna, Bologna, Italy. ${ }^{2}$ Desmond Tutu TB Centre, Dept of Paediatrics and Child Health, Faculty of Medicine and Health Sciences, Stellenbosch University, Cape Town, South Africa. ${ }^{3}$ Fondazione S. Maugeri, IRCCS, Tradate, Italy. ${ }^{4}$ Public Health Consulting Group, Lugano, Switzerland. ${ }^{5}$ Médecins Sans Frontières, Paris, France. ${ }^{6}$ Children's Hospital at Westmead and the Centre for Research Excellence in Tuberculosis, University of Sydney, Sydney, Australia. ${ }^{7}$ MDR/XDR TB Unit, Tshepong Hospital Klerksdorp, Klerksdorp, South Africa. ${ }^{8}$ MDR-TB Unit, Tuberculosis Division, International Union against Tuberculosis and Lung Disease (The Union), Paris, France. ${ }^{9}$ Pneumology Dept, Hospital General de Gran Canaria “Dr Negrin", Las Palmas de Gran Canaria, Spain. ${ }^{10}$ Médecins Sans Frontières, Mumbai, India. ${ }^{11}$ Médecins Sans Frontières, Moscow, Russian Federation. ${ }^{12}$ Médecins Sans Frontières, London, UK. ${ }^{13}$ Republican TB Dispensary, Grozny, Chechen Republic, Russian Federation. ${ }^{14}$ Armenian National Tuberculosis Control Centre, Yerevan, Armenia. ${ }^{15}$ Médecins Sans Frontières, Yerevan, Armenia. ${ }^{16}$ National Center for Tuberculosis and Lung Diseases, Tbilisi, Georgia. ${ }^{17}$ Médecins Sans Frontières, Tbilisi, Georgia. ${ }^{18}$ Paediatric Highly Intensive Care Unit, Dept of Pathophysiology and Transplantation, Università degli Studi di Milano, Fondazione IRCCS Ca' Granda Ospedale Maggiore Policlinico, Milan, Italy. ${ }^{19}$ University Dept of Infectious and Tropical Diseases, University of Brescia and Brescia Spedali Civili General Hospital, Brescia, Italy. ${ }^{20}$ Harvard Medical School, Dept of Global Health and Social Medicine, Boston, MA, USA. ${ }^{21}$ These authors contributed equally.

Correspondence: Giovanni Battista Migliori, Fondazione S. Maugeri, Care and Research Institute, Via Roncaccio 16, 21049, Tradate, Italy. E-mail: giovannibattista.migliori@fsm.it

Received: April 072016 | Accepted after revision: May 092016 | First published online: June 232016

This article is one of a small annual allocation selected for open access publication at the discretion of the editor.

Conflict of interest: None declared.

Acknowledgements: The authors wish to thank Gunar Günther (Dept of Medicine, University of Namibia, School of Medicine, Windhoek, Namibia), Zarir F. Udwadia (Dept of Respiratory Medicine, P.D. Hinduja National Hospital and Medical Research Centre, Mumbai, India) and Charles Ssonko (Médecins Sans Frontières, Mbabane, Swaziland) for providing information on their cases. The authors alone are responsible for the views expressed in this article and they do not necessarily represent the decisions and policies of their institutions.

\section{References}

1 Jenkins HE, Tolman AW, Yuen CM, et al. Incidence of multidrug-resistant tuberculosis disease in children: systematic review and global estimates. Lancet 2014; 383: 1572-1579.

2 Migliori GB, Sotgiu G, Gandhi NR, et al. Drug resistance beyond extensively drug-resistant tuberculosis: individual patient data meta-analysis. Eur Respir J 2013; 42: 169-179.

3 Diel R, Vandeputte J, de Vries G, et al. Costs of tuberculosis disease in the European Union: a systematic analysis and cost calculation. Eur Respir J 2014; 43: 554-565.

4 Seddon JA, Furin JJ, Gale M, et al. Caring for children with drug-resistant tuberculosis: practice-based recommendations. Am J Respir Crit Care Med 2012; 186: 953-964. 
5 World Health Organization. Guidance for National Tuberculosis Programmes on the Management of Tuberculosis in Children. 2nd Edn. WHO/HTM/TB/2014.03. Geneva, World Health Organization, 2014. http://apps.who.int/ medicinedocs/documents/s21535en/s21535en.pdf

6 Bonnet $\mathrm{M}$, Bastard $\mathrm{M}$, du Cros $\mathrm{P}$, et al. Identification of patients who could benefit from bedaquiline or delamanid: a multisite MDR-TB cohort study. Int J Tuberc Lung Dis 2016; 20: 177-186.

7 Pontali E, Sotgiu G, D'Ambrosio L, et al. Bedaquiline and multidrug-resistant tuberculosis: a systematic and critical analysis of the evidence. Eur Respir J 2016; 47: 394-402.

8 Esposito S, D'Ambrosio L, Tadolini M, et al. ERS/WHO Tuberculosis Consilium assistance with extensively drug-resistant tuberculosis management in a child: case study of compassionate delamanid use. Eur Respir J 2014; 44: $811-815$.

9 Tiberi S, De Lorenzo S, Centis R, et al. Bedaquiline in MDR/XDR-TB cases: first experience on compassionate use. Eur Respir J 2014; 43: 289-292.

10 Guglielmetti L, Le Dû D, Jachym M, et al. Compassionate use of bedaquiline for the treatment of multidrug-resistant and extensively drug-resistant tuberculosis: interim analysis of a French cohort. Clin Infect Dis 2015; 60: $188-194$.

11 Lessem E, Cox H, Daniels C, et al. Access to new medications for the treatment of drug-resistant tuberculosis: patient, provider and community perspectives. Int J Infect Dis 2015; 32: 56-60.

12 World Health Organization. Companion Handbook to the WHO Guidelines for the Programmatic Management of Drug-resistant Tuberculosis. WHO/HTM/TB/2014.11. Geneva, World Health Organization, 2014. http://apps. who.int/iris/bitstream/10665/130918/1/9789241548809_eng.pdf

13 Lachâtre M, Rioux C, Dû DL, et al. Bedaquiline plus delamanid for XDR tuberculosis. Lancet Infect Dis 2016; 16: 294.

14 Tadolini M, Lingtsang RD, Tiberi S, et al. First case of extensively drug-resistant tuberculosis treated with both delamanid and bedaquiline. Eur Respir J 2016; in press [DOI: 10.1183/13993003.00637-2016].

15 Matteelli A, D'Ambrosio L, Centis R, et al. Compassionate and optimum use of new tuberculosis drugs. Lancet Infect Dis 2015; 15: 1131-1132.

16 Nachman S, Ahmed A, Amanullah F, et al. Towards early inclusion of children in tuberculosis drugs trials: a consensus statement. Lancet Infect Dis 2015; 15: 711-720.

\title{
Variation in policy and practice of adolescent tuberculosis management in the WHO European Region
}

\author{
To the Editor:
}

Tuberculosis (TB) is a major public health concern worldwide. The World Health Organization (WHO) estimates that 9.6 million people fell ill with $\mathrm{TB}$ and 1.5 million people died of $\mathrm{TB}$ in 2014 . Of an estimated total of 1 million children with TB, 140000 children died [1]. The risk of progression to TB is highest for children $<5$ years of age. There is a comparatively low risk for those 5-10 years of age, after which the risk of progression to TB increases at the onset of puberty to an adult level of 5-10\% [2] as well as the chance of developing an adult-type TB accompanied by increased infectiousness [3]. However, adolescents form a particularly vulnerable group with psychosocial challenges distinct from adults and the usual challenges of transition from paediatric to adult health service provision. Puberty may affect health behaviour and have a significant impact on therapy compliance [4], requiring specific approaches usually not provided by paediatric or adult health services [5].

The current guidelines for childhood TB cover children from 0 to 14 years of age but do not specifically deal with adolescents aged 10-18 years $[5,6]$. WHO surveillance guidelines recommend to record and report two age bands (0-4 years and 5-14 years) [5], and thus specific epidemiological information on adolescents is lacking. To gain more insight in TB management among adolescents, the WHO European Regional Task Force on childhood TB conducted a survey among the 53 member states of the WHO European Region [7]. In January 2015, all national TB programme managers and designated childhood TB focal points were asked to provide information on the existence and nature of policies and practical management of adolescents with TB in their country. Responses received by April 30, 2015 were analysed using Excel 2010 (Microsoft, Redmond, WA, USA). 28 (53\%) countries responded to the first part of the questionnaire on policy and 20 countries also completed the questions on management of adolescents with TB. The relevant results are summarised in table 1. 